\title{
Una caja de herramientas para medir el universo de protestas en México
}

\author{
Roberto Holguín Carrillo
}

\begin{abstract}
Resumen
El análisis de eventos de protesta es una opción viable y robusta para recuperar, analizar e interpretar datos que ofrecen un panorama general de la trayectoria y dinámica de manifestaciones. El Laboratorio de Análisis de Organizaciones y Movimientos Sociales (LAOMS) es uno de los espacios donde se desarrolla esta metodología en México, y para su aplicación se ha implementado una serie de procesos que involucran el uso de tecnologías de información. Mediante LimeSurvey, un software libre para realizar todo tipo de encuestas en línea, el LAOMs puede generar una base de datos sobre protestas. El software permite el diseño de encuestas, la gestión de encuestados, exportación de registros y conteos rápidos, lo que posibilita recuperar y codificar información de forma ágil y remota. LimeSurvey es una opción asequible y de gran funcionalidad que puede ser adaptada a diversos proyectos e intereses de investigación.
\end{abstract}

Palabras clave: análisis de eventos de protesta, movimientos sociales, metodología, Limesurvey, encuestas.

\section{A toolbox for meAsURING PROTESTS in MEXICO}

\begin{abstract}
Protest event analysis offers a viable and robust option to collect, analyze and interpret data about the dynamic and trajectory of protests from a general perspective. The Laboratory of Analysis of Organizations and Social Movements (LAOMS) is one of the places where this methodology is developed in Mexico, and for its application we have implemented a series of processes that involve the use of information technologies. With LimeSurvey, a free and open source software to apply surveys online, LAoms can generate a database of protests. The software features survey design, respondent management, registry exports and statistics, which allow us to collect and code data remotely and easily. Limesurvey is an affordable solution with great functionality, which can be adapted for different research projects and interests.
\end{abstract}

Keywords: protest event analysis, social movements, methodology, Limesurvey, surveys.

Dol: http://doi.org/10.22201/codeic.16076079e.2020.v21n3.a2 


\section{Roberto Holguín Carrillo}

Roberto Holguín es egresado de la Licenciatura en Historia, de la Facultad de Filosofía y Letras de la unam. Se desempeña como responsable de sistemas en el Consejo Mexicano de Ciencias Sociales (COMECSO) y como desarrollador principal en el Laboratorio de Análisis de Organizaciones y Movimientos Sociales (LAOMS).

Sus intereses de investigación son las rebeliones del siglo xix mexicano y los movimientos sociales y revolucionarios del siglo xx y xxı. Entusiasta del uso de herramientas digitales en las humanidades y ciencias sociales para el análisis, visualización y difusión de datos sobre procesos políticos y sociales contemporáneos. 


\section{Introducción}

En México, las protestas son y serán parte del centro de la atención pública. A un país atravesado por múltiples dificultades sociales, con reclamos y agravios tan variados como sus habitantes, le es imposible dejar de traducir en protestas su malestar de forma cotidiana. Éstas recuperan, mediante actos organizativos, la noción de lo colectivo como fundamento para la resolución de problemas apremiantes. Algunas formas de protesta, las marchas, por ejemplo, evidencian esta idea muy claramente: se toma la calle, espacio público inevitable, para recordar que estamos todos involucrados y que son pocos los problemas que se originan y resuelven estrictamente en solitario, que necesitamos ayuda.

De ello parte la importancia de las protestas, que buscan visibilizar agravios, ofrecer diagnósticos y establecer interacciones constantes entre los actores involucrados para avanzar en su resolución. Se puede entender a las protestas y a las interacciones que promueven como ingredientes que conforman los movimientos sociales ${ }^{1}$. Dan cuenta de nuestras libertades civiles, bordean los espacios institucionales y legales, las vías de la política partidista y de los medios periodísticos. Ofrecen un recurso a aquellos que no encuentran un lugar en estos espacios o que han recurrido a ellos sin obtener éxito. Es, entonces, necesario tener una idea general de cómo se desarrollan las protestas, su dinámica y trayectoria para entender nuestras posibilidades de cambio como sociedad.

Desde hace una década, el Laboratorio de Análisis de Organizaciones y Movimientos Sociales (LAOMS) de la Universidad Nacional Autónoma de México (UNAM) ha emprendido la laboriosa tarea de contribuir en la formación de aquella idea general sobre el universo de protestas en México. Este artículo aborda algunos de los enredos digitales que involucró; es decir, los desafíos de implementación, diseño y gestión involucrados para aprovechar las tecnologías de información disponibles con el fin de aproximar una medición del universo de organizaciones y movimientos sociales.

\section{LAOMS y el análisis de eventos de protesta}

Algunos estudios que sirven como introducción para esta perspectiva de los movimientos sociales son Cadena Roa (2016) e Inclán Oseguera (2017), que se incluyen en las referencias de este artículo.
El LAOMs es un espacio interdisciplinario de análisis y seguimiento de protestas en México creado durante 2009 en el Centro de Investigaciones Interdisciplinarias en Ciencias y Humanidades ( $\mathrm{CEIICH}$ ) de la unam. Sus objetivos iniciales buscaron estrechar la colaboración entre activistas y miembros de organizaciones de los movimientos sociales con el sector académico, para que cada uno desde su trinchera contara con más información, estableciera puntos de dialogo y tuviera mejores herramientas para lograr sus actividades (Cadena y Falleti, 2009). Desde su surgimiento, el LAOms promueve encuentros de este tipo, así como de estudiosos de los movimientos sociales dentro y fuera del país, y de miembros de laboratorios y observatorios similares principalmente de América Latina. como grupo de investigación ha producido artículos, informes, tesis y ponencias 
${ }^{2}$ El análisis de eventos de protesta permite valerse de otras fuentes, como reportes policiacos, reportes de líneas telefónicas de atención ciudadana, redes sociales, entre otras.

${ }^{3}$ Se puede considerar que los campos representan las interacciones constantes entre demandantes y demandados que conforman los movimientos sociales (por ejemplo, lo que habitualmente se conoce como el movimiento campesino, estudiantil, feminista) (Snow, 2004), pero ponen el acento en la heterogeneidad de los actores que los componen y con los que coexisten, los objetivos que persiguen y el potencial de movilización que tienen (Cadena Roa, 2016). que se han presentado en congresos nacionales e internacionales, algunos de los cuales pueden consultarse aquí.

Otro producto del LAOMS es la elaboración de una metodología y herramientas acompañantes específicas para, como decíamos, medir el universo de protestas. Aquí conviene precisar el concepto de medición ya que es muy válido preguntarse si la protesta es algo que se mide. En definitiva, no tiene las características que se ajustan a una unidad de medida convencional: no tiene las propiedades de equivalencia, magnitud y ordenación de las unidades usadas en astronomía, por ejemplo. Sin embargo, como las estrellas, las protestas tienen coordenadas. Son sucesos que transcurren en un tiempo y un lugar específico, es decir, algo que puede fijarse en el devenir histórico. Este punto temporal, que llamamos evento, es un recurso de relativa antigüedad en las ciencias sociales para realizar exploraciones en períodos considerablemente largos. De tal forma que, inicialmente, medir en este contexto implica sencillamente explorar o dimensionar.

Para delimitar la investigación de la protesta al concepto de evento le hemos añadido diversas características. Además de su ubicación temporal y geográfica, lo definimos principalmente como acción colectiva, es decir, como una acción concertada por un grupo de individuos que presenta demandas a otros y que se vale de una serie de repertorios de acción para visibilizar sus peticiones. En el LAOMs estas rutinas se consideran como actividades que escapan de las vías legales o institucionales e introducen incertidumbre en las actividades de los demás mediante acciones disruptivas como bloqueos, plantones, marchas, huelgas, entre otras (Tarrow, 2012; Tilly, 1986).

En el estudio de movimientos sociales, esta metodología es una variante de investigación conocida como análisis de eventos de protesta (AEP). Su aplicación supone una definición de criterios para acceder y extraer información de fuentes documentales, principalmente periodísticas². Éstos definen qué tipo de información se obtiene, cómo hacerlo y cómo organizarla. A este proceso se le llama codificación, y usualmente es elaborada por una persona frente a una computadora conocida como capturista. La labor de codificación se traduce en una base de datos en la que cada registro es un evento de protesta que incluye la información recabada, de acuerdo con los criterios definidos.

Actualmente el LAOMs considera o recupera diez campos de información: fecha, lugar, actor, campo de los movimientos sociales o de la acción colectiva ${ }^{3}$, demandas, repertorios de protesta, a quién se piden las demandas, origen de los agravios, alcance territorial y respuestas a la protesta. Todos los registros de nuestra base de datos cuentan con al menos los seis primeros campos. Por ejemplo, un capturista encuentra un evento de protesta en el periódico, registra la fecha (supongamos 19 de mayo de 2012), el actor (YoSoy132), el lugar (Zócalo en la Ciudad de México), el campo de los movimientos sociales (de estudiantes), las demandas (democratización de medios) y su repertorio 
(manifestación o marcha). Todo esto se añade a nuestra base de datos como un registro independiente.

El conteo de los eventos de protesta capturados permite trazar series temporales, para analizar el número de protestas a lo largo de varios años y modificar el análisis de acuerdo con actores, lugares, demandas y demás datos disponibles (ver figura 1). Este tipo de análisis permite el fácil intercambio entre visiones generales y particulares, entre análisis macro y micro. Así, es posible analizar primero todas las protestas de 2012 y después filtrar los datos para examinar solamente las protestas estudiantiles por mes o incluso por día.

El éxito del AEP se apoya en gran medida en dicho intercambio y de allí una buena parte de su riqueza. El principal reto que involucra, como refiere Roberto Franzosi (2004), es el proceso de conversión de "palabras a números", el traducir información textual en datos cuantificables. Implica una cantidad de recursos considerables, instrumentación teórica y operativa, y largos procesos de depuración de datos que están sujetos constantemente a errores y sesgos, tanto de la fuente informativa como de la labor de investigación.

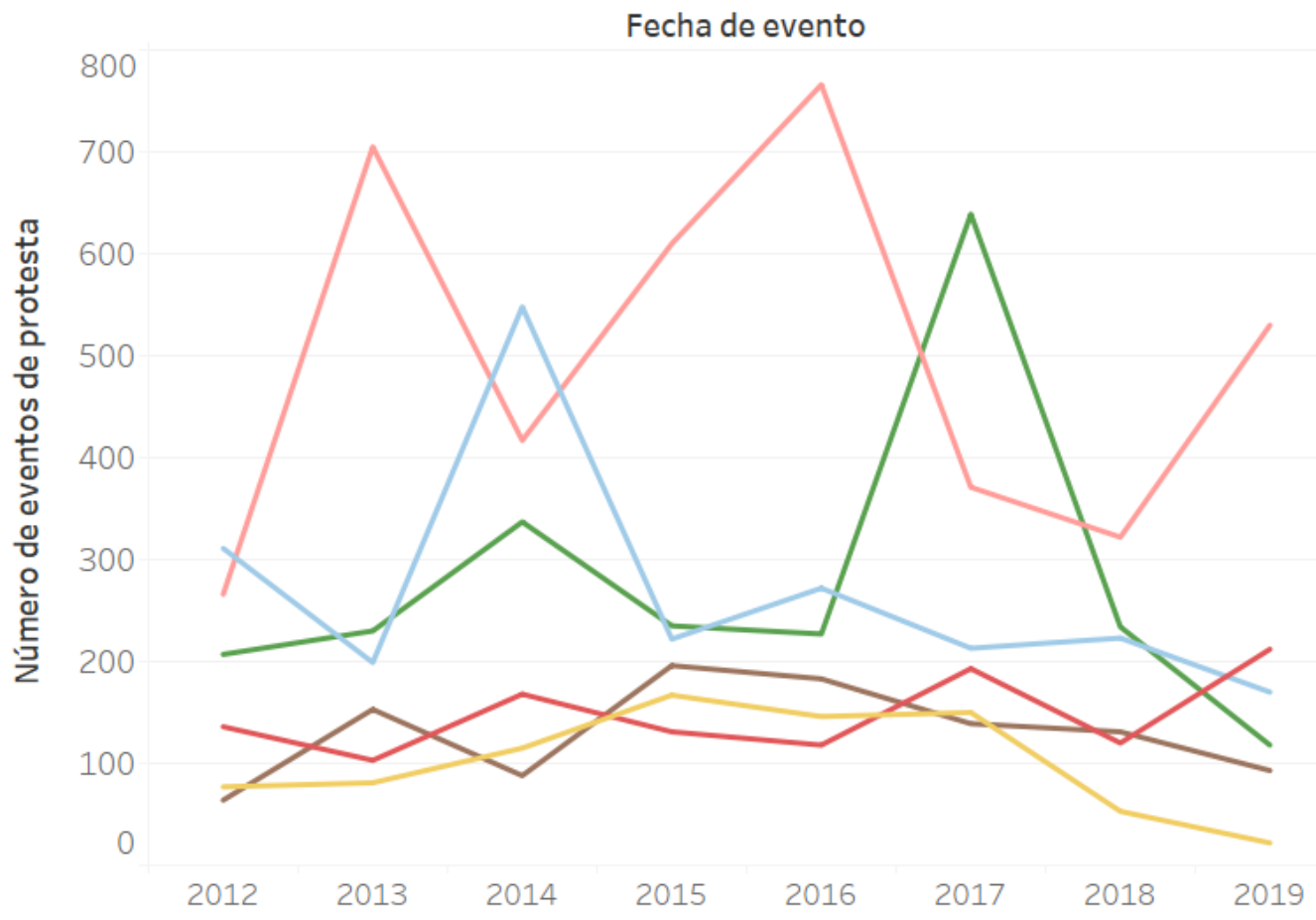

Figura 1. Volumen de participación de principales tipos de actor por año. Fuente: elaboración propia con datos del LAOMS. Datos preliminares.
Tipos de actor

Afectados y víctimas de la inseguridad pública y violencia

Campesinos

Educadores, maestros

Estudiantes

Sindicatos del sector público

Vecinos, pobladores, habitantes 
Pese a los retos involucrados, las ventajas del AEP destacan por el sustento empírico y documental que ofrece, debido al tratamiento equitativo y ordenado de la información disponible. Sin importar de qué tema sea un evento, si ocurre bajo alguno de los repertorios de protesta analizados, se cuenta como cualquier otro. Esto es relevante porque contribuye a la elaboración y soporte de teorías que explican de forma general características y dinámicas de los movimientos sociales, así como a la formación del respaldo empírico necesario para contextualizar y dimensionar movimientos sociales particulares, es decir, para estrechar la "brecha entre la teoría y los datos" (Koopmans y Rucht, 1999).

Aún podemos encontrar en varios estudios escaso soporte documental en la formulación de afirmaciones generales o bien la sobrepoblación de estudios de caso demasiado particulares. No por ello se debe pensar erróneamente que esta metodología se presenta, como la "que acabará con todas las metodologías", portadora de la única verdad e imposible de conciliar con las demás. Más bien, el AEP opera como una metodología complementaria, que permite conjuntar enfoques cualitativos, cuantitativos y el desarrollo teórico de diversas disciplinas de las ciencias sociales, en torno a los movimientos sociales (Oliver, Cadena-Roa y Strawn, 2003).

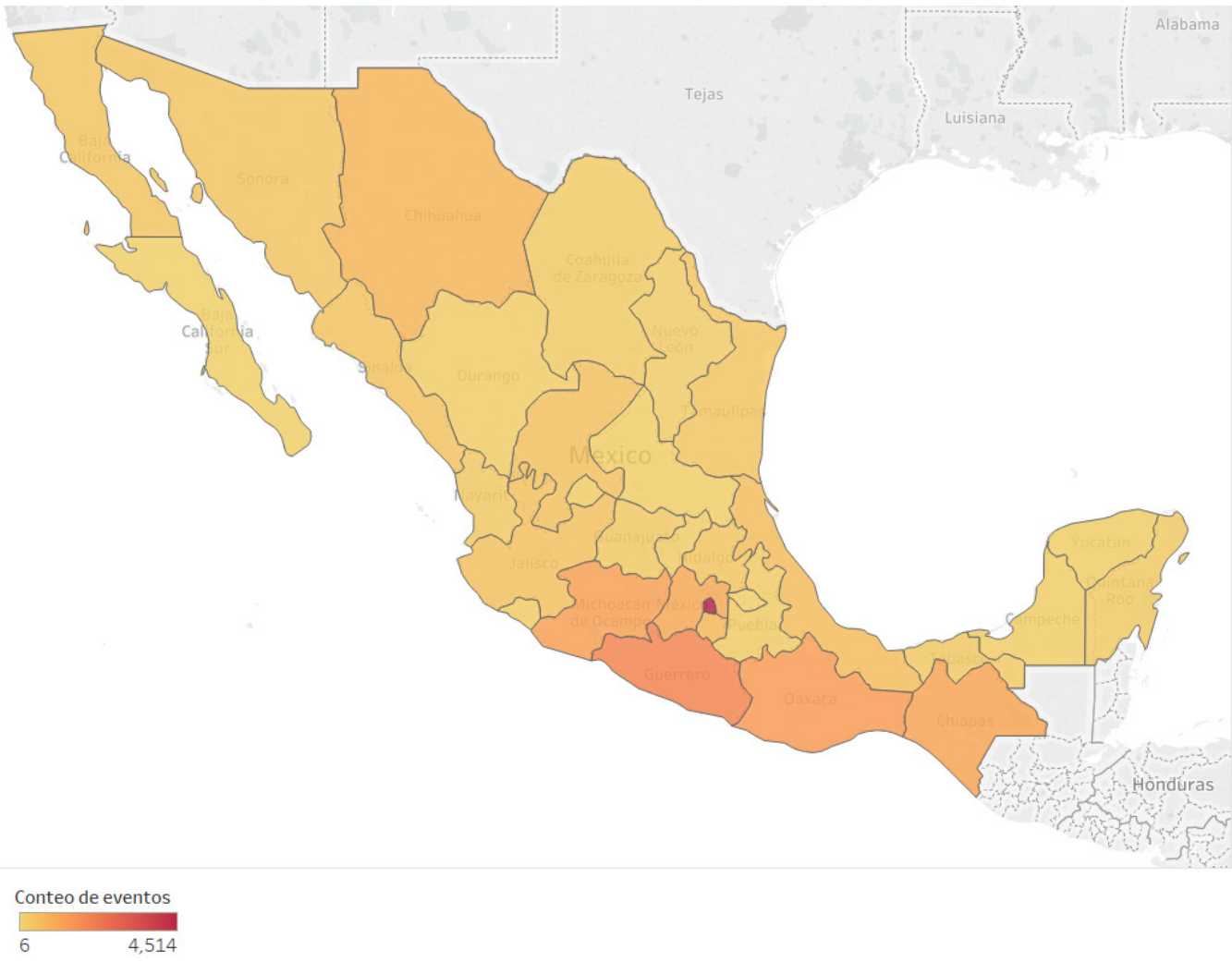

Actualmente, el LAOMs cuenta con más de 17 mil registros sobre protestas que van del 2012 a la fecha y que cubren todo el país (ver figura 2). El AEP nos ha 
${ }^{4}$ Las palabras clave corresponden en su mayoría a los repertorios de protesta utilizados con mayor frecuencia, algunos de los cuales son mencionados brevemente en el apartado anterior.

${ }^{5}$ Principalmente la posibilidad de utilizar operadores booleanos para identificar palabras tanto en el encabezado como en el cuerpo de la nota. Para más información sobre este tipo de operadores, puede consultarse el documento de la Biblioteca Daniel Cosío Villegas de El Colegio de México "Estrategias de búsqueda", disponible en http://biblio. colmex.mx/curso investigacion documental/Estrategias $\% 20$ de\%20b\%C3\%BAsqueda.pdf permitido, entre otras cosas, analizar el tejido organizacional que subyace en las protestas, sus actores principales, así como los marginales y emergentes (Cadena Roa, 2016; Holguín Carrillo; 2016; Hernández Díaz; 2019). También se han explorado algunas de las relaciones que existen entre el desarrollo de la política institucional (como el de las elecciones y la agenda legislativa) y el terreno de las protestas, entre la coyuntura política y la aparición de oleadas de protesta (Cadena y Urbina, 2016). Asimismo, el AEP ha facilitado trazar un mapa de las temáticas de la protesta y analizar en qué contextos ciertas demandas tienen mayor atención que otras (González y Zirión, 2019).

\section{Dinámica de selección y codificación de eventos de protesta}

En el proceso de selección y codificación de información del LAOMs conviven técnicas de análisis humano y de utilización de herramientas digitales que permiten la implementación efectiva de nuestra metodología. El primer paso es la recopilación de fuentes útiles para recuperar eventos de protesta. Esta labor se realiza con la ayuda de los índices del buscador Google generados para el periódico de circulación nacional mexicano La Jornada. Por el momento, es el único periódico, ya que hay una serie de retos y recursos involucrados en la inclusión de diversas fuentes que desafortunadamente nos impiden recurrir a más medios. Sin embargo, este diario aporta de forma consistente una buena cantidad de información sobre protestas en el país, a pesar de su sesgo hacia la cobertura de eventos principalmente de la ciudad de México (Hernández Díaz, 2019).

La búsqueda en los índices se realiza medianteel uso de una serie de palabras clave $^{4}$ que son introducidas en un solo intento a través de las funcionalidades avanzadas de búsqueda de Google ${ }^{5}$. El buscador arroja notas relevantes, lo cual agiliza el proceso de selección. Además, contamos con una herramienta elaborada en casa que se vale del Motor de búsqueda personalizado, también de Google, para precisar las búsquedas, además de superar algunas limitaciones del buscador en línea (principalmente el límite de número de palabras). Esta herramienta nos permite hacer búsquedas por cada repertorio de protesta o verbos comúnmente asociados con ella. Por ejemplo, el verbo "marcha", puede utilizarse como un repertorio, pero también en construcciones como "poner en marcha", "estuvo en marcha" y similares. Nuestra metodología permite añadir un sin número de construcciones verbales vinculadas con protestas y descartar todas las que no están asociadas, para después agrupar todas las búsquedas en una tabla para su visualización y respaldo.

Durante el trabajo de selección mediante Google, una persona certifica que las notas recuperadas cumplieron con las particularidades de un evento de protesta mencionadas anteriormente. Una vez que pasan este filtro, se incorporan a una carpeta en Dropbox, para después ser sincronizadas con nuestro servidor. La codificación de estas notas se logra a través de la interacción 
${ }^{6} \mathrm{El}$ software libre funciona bajo distintos modelos de licencias, las cuales respaldan legalmente el que pueda permanecer libre. En el caso de LimeSurvey, utiliza la popular licencia GPL (General Public License), elaborada por la Free Software Foundation, que permite publicar modificaciones al código siempre y cuando permanezca abierto a los receptores. Más detalles pueden consultarse en la siguiente liga: https://www.gnu.org/licenses/ gpl-faq.html.

${ }^{7}$ La lista completa de tipos de preguntas puede consultarse en el Manual de LimeSurvey en https://manual.limesurvey.org/ Question types/es. de un capturista con un programa disponible vía internet y alojado en nuestro servidor que utiliza LimeSurvey, un software libre originalmente destinado al diseño y levantamiento de encuestas en línea.

Para la construcción de la base de datos, en el LAOMs recurrimos inicialmente a un diseño de software a medida, es decir, el código informático involucrado fue elaborado desde cero de acuerdo con nuestros requisitos específicos. Pese a la libertad de posibilidades y personalización que esto permitía, el programa requería de costosas intervenciones para la atención de requisitos nuevos o modificaciones. Al ser de código cerrado, en oposición con el software libre, cuyo código es público para su análisis y modificación, el desarrollador inicial era el único que conocía el código a detalle y era capaz de realizar modificaciones. Esto se agravó cuando se acumularon datos abundantes, ya que las modificaciones necesarias no sólo involucraban la captura sino también su análisis. Es por eso que se consideró la implementación de una nueva plataforma de captura basada en el ya mencionado LimeSurvey.

\section{Las características de LimeSurvey}

LimeSurvey, al ser un programa en línea, permitió retomar la dinámica de captura con rapidez, además de que es fácil de utilizar, pues no necesita un software o hardware especializado. Implementarlo favoreció un diseño de base de datos más acorde con nuestras necesidades de análisis, simplificar nuestras preguntas de investigación y agilizar el proceso de captura de información.

A pesar de ser un programa para realizar encuestas, logramos adaptarlo con relativa facilidad pues pudimos crear un formulario de entrada de información, que después consolidaba los registros introducidos en una tabla con todos los datos (ver figura 3). En otras palabras, nos permitió traducir las preguntas — que le hacíamos a los periódicos en torno a las protestas - en una base de datos de donde podían extraerse conteos, resúmenes y gráficas.

Las características de LimeSurvey para el diseño de formularios son variadas. Es posible añadir hasta 29 tipos de preguntas, las cuales van desde campos que admiten entrada numérica, de texto, de opción múltiple o única. ${ }^{7}$ Así, se permitió conjuntar el uso de categorías para agrupar la información con una más novedosa. Un ejemplo es la codificación que hacemos de las demandas. Si encontramos una protesta de estudiantes que piden la "aparición con vida de los 43 normalistas", en la interfaz de captura introducimos la demanda textual, tal cual viene en la nota periodística, y después la agrupamos en las clasificaciones que hemos diseñado para distintas demandas, en este caso, la clasificamos en el grupo de demandas en torno a Justicia. Este tipo de concentraciones nos permite analizar de forma general las demandas, es decir, examinar el peso que tiene cada una de las clasificaciones en el total de las protestas desde el mismo módulo de estadísticas incluido en LimeSurvey (ver figura 4). 
* 2. Fecha periódico

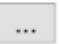

Formato: $d d / \mathrm{mm} / \mathrm{yyyy}$

* 3. Fecha evento

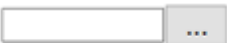

Formato: $d d / \mathrm{mm} / \mathrm{y} y y y$

\section{¿Es un EP que dura más de un día?}

\section{Si}

\section{* 4. Actor}

Seleccione una de las siguientes opciones

OMS - Organización de los Movimientos Sociales

\section{Registrar organizaciones (OMS)}

Figura 3. Una sección de la interfaz de captura de eventos de protesta. Fuente: interfaz de captura en http://laoms.org.

Figura 4. Gráfico de barras que muestra el volumen de demandas de acuerdo con su clasificación. Fuente: elaboración propia con datos del LAOMS. Datos preliminares.
- 1. Abusos de autoridades en eventos de protesta (280)

-2. Bienes y servicios (especie) / efectivo y presupuesto (dinero) (3059)

- 3. Demandas a la administración pública (733)

= 4. Justicia (procuración e impartición de justicia) (4019)

-5. Laborales (3121)

- 6. Protección de derechos de grupos vulnerables (613) 7. Reformas a leyes y políticas gubernamentales (2428)

- 8. Defensa del espacio. los recursos y la cultura (898)

= 9. Solidaridad (106)

- 10. Apertura de canales de diálogo y negociación, demandas de transparencia... (1931)

= 11. Indeterminado (18)

- Sin respuesta (12) No completada o No mostrada (0) 
Otra característica especialmente relevante es la definición de una lógica de encuesta, la cual establece relaciones entre pregunta y pregunta, y determina si se debe mostrar o no alguna de ellas. El registro de los lugares en los que ocurre la protesta es un ejemplo de esto. Sería poco eficiente utilizar campos de texto abiertos (en los que se puede introducir cualquier texto) para definir lugares que sabemos con anticipación, como los estados y municipios de México. De tal forma que hemos colocado a estos en listas que se despliegan con una serie de opciones acotadas. Al seleccionar, por ejemplo, la Ciudad de México, solamente se despliegan las alcaldías que lo componen. De esta forma, nuestros datos son consistentes y la captura es más ágil porque la información disponible está incluida en el mismo formulario de entrada. También se destaca el módulo de estadísticas, debido a sus funcionalidades de exportación de la información para su análisis.

Por supuesto que las características del programa en conjunto exceden las mencionadas y pueden revisarse extensivamente en la página del proyecto, basta con destacar en este punto que LimeSurvey nos permitió resolver la complejidad de la captura de información de acuerdo con nuestros criterios a pesar de que, a primera vista, el propósito del programa no parecía emparentarse del todo con el nuestro.

\section{¿Por qué usamos LimeSurvey?}

La elección de utilizar LimeSurvey para la captura de nuestros datos partió de una investigación para identificar el método más conveniente ya que cada día hay una amplia oferta disponible de herramientas para el diseño de formularios, como los formularios de Google o Google Forms y SurveyMonkey. LimeSurvey resultó la mejor opción para el LAOMs por sus múltiples características y costo relativamente bajo. Para su funcionamiento contamos con un servidor económico (alrededor de 6 dólares [USD] al mes), que también usamos para labores de difusión. Su instalación, así como su uso, es sencilla pues no requiere capacitación extensa para navegar en sus opciones. Es posible acceder a demostraciones (demos) en línea, o bien, adquirir una versión que evita la labor técnica de instalación y su configuración inicial puede realizarse en pocos minutos, aunque, reduce las posibilidades avanzadas de personalización.

\section{Conclusiones: ¿qué aprendimos?}

Es importante hablar de la experiencia del LAOMs dado que los desafíos y resultados presentados pueden tener coincidencias con proyectos y objetivos de otros grupos de investigación, al menos de forma general. Si se piensa en la noción básica de un formulario, que puede recabar información de modo sistemático, LimeSurvey será útil para ser aplicado en otros proyectos y atender otras preguntas de investigación. 
Lo anterior también nos llevó a revalorizar la aproximación al uso de software. Es fácil caer en soluciones apresuradas, por eso es importante investigar a fondo las herramientas disponibles y valorar si cumplen con los objetivos, si son asequibles, si sus carencias pueden ser complementadas con otras y si es necesario emprender un proyecto para elaborar software nuevo.

Una de las pautas técnicas del LaOMs ha sido pensar en diseños modulares de manera que el trabajo pueda hacerse mediante la conexión de distintas herramientas. No es necesario inventar nuevos programas de estadística cuando existen instrumentos muy logrados, algunos de forma gratuita.

También es importante que las personas encargadas del análisis de los datos se involucren en el diseño y en la implementación de herramientas técnicas. Es común que los proyectos deleguen toda la tarea al personal técnico o de informática y al final los datos no cumplan con los propósitos de la investigación. De igual manera, el diseño de las bases de datos resulta importante y susceptible a este tipo de colaboración, para ello se recomiendan herramientas en las que se puedan hacer pruebas fácilmente modificables de los resultados esperados o de las visualizaciones necesarias para la entrada y salida de la información, comúnmente conocidas como de prototipado, de las cuales existen varias opciones. ${ }^{8}$

Finalmente, es importante destacar los múltiples efectos positivos que tiene el utilizar software libre para una tarea de investigación de esta índole y para el trabajo de instituciones de enseñanza pública como la unAm. Este tipo de software conduce hacia procesos de aprendizaje interactivos y promueve una vieja costumbre en la ciencia y la formación del conocimiento: la colaboración y edificación a partir del trabajo de los demás.

\section{Referencias}

* Cadena Roa, Jorge (2016), Las organizaciones de los movimientos sociales y los movimientos sociales en México, 2000-2014: Friedrich Ebert Stiftung. http:// laoms.org/organizaciones-de-los-movimientos-sociales-mexico-2000-2014/.

${ }^{8}$ Puede conocerse más sobre este enfoque y sus herramientas en el documento Prototipado elaborado por Walter Maner y disponible en español en este enlace: http://www.sidar.org/ recur/desdi/traduc/es/visitable/ maner/Prototipado.htm
- Cadena Roa, Jorge y Valeria Falleti. (2009). El Laboratorio de Análisis de Organizaciones y Movimientos Sociales (LAOMS). Objetivos y actividades iniciales. En Amozurrutia de María y Campos, José Antonio, Ricardo Lino Mansilla Corona y Javier Álvaro Matus Pacheco (Coords.), Jornadas Anuales de Investigación, 2009 (CEIICH-UNAM, pp. 23-33). https://www.academia.edu/1425860/El Laboratorio de An\%C3\%A1lisis de Organizaciones y Movimientos Sociales LAOMS. Objetivos y actividades iniciales. 
* Cadena J. \& Urbina, G. (Eds.). (2015). Informe anual 2013. Un año de reformas y de movilización social. Proyecto de Ciencia Básica Desempeño Organizacional: organizaciones de la sociedad civil (osc), organizaciones de los movimientos sociales (омs) y acción colectiva. México, D. F., México: Centro de Estudios e Centro de Investigaciones Interdisciplinarias en Ciencias y Humanidades-Universidad Nacional Autónoma de México [no publicado].

* Franzosi, Roberto (2004). From words to numbers: narrative, data, and social science. Cambridge University Press.

* González Arroyo, Karina Guadalupe y Sofía Zirión Martínez. (2019). 2012-2016. Enrique Peña Nieto y los años de la inconformidad social: Un análisis desde los eventos de protesta. En Juan Carlos Ruiz Guadalajara y Gustavo A. Urbina Cortés (Coords.), Las ciencias sociales y la agenda nacional. Vol. II. Acción colectiva, movimientos sociales, (pp. 217-233). Consejo Mexicano de Ciencias Sociales. https://www.comecso.com/ ciencias-sociales-agenda-nacional/cs/article/view/357.

* Hernández Díaz, Daniel (2019). Análisis de redes aplicado a coaliciones de eventos de protesta reportadas por el diario La Jornada (octubre 2012 a diciembre 2016) [tesis para obtener el título de Licenciado en Matemáticas Aplicadas y Computación, unAm, Facultad de Estudios Superiores Acatlán]. https://github.com/DanielHD/ Tesis.

* Holguín Carrillo, Roberto. (2016). ¿Quién es quién entre los actores emergentes de las protestas y movimientos sociales en México?. En Memorias del 5to Congreso Nacional de Ciencias Sociales, comecso, Eje 9, marzo 2016, pp. 1195-1213. https://www. academia.edu/24175606/ Qui\%C3\%A9n es qui\%C3\%A9n entre los actores emergentes de las protestas y movimientos sociales en M\%C3\%A9xico.

* Holguín Carrillo, Roberto. (2018). Variables de la dinámica de la represión e interlocución en las protestas en México (2013-2016). En Manuel Alcántara, Mercedes García Montero, Mercedes y Francisco Sánchez López (Coords.), Movimientos Sociales: Memoria del 56. ${ }^{\circ}$ Congreso Internacional de Americanistas (pp. 339-350). Ediciones Universidad de Salamanca. https://play.google.com/books/ reader?id=VbNIDWAAQBA].

* Inclán Oseguera, María de la Luz (2017). A la sombra de Sidney Tarrow, Conceptos básicos para el estudio de los movimientos de protesta. Política y Gobierno, xxIv(1), 1er semestre de 2017,189-212.

* Koopmans Ruud y Dietr Rucht. (1999) Protest Event Analysis-Where to Now? Mobilization, 4(2), 123-130.

* Oliver, Pamela E., Cadena-Roa, J. \& Strawn, Kelley D. (2003). Emerging trends in the study of protest and social movements. Research in Political Sociology. Political Sociology for the 21st Century, 12, 213-244.

- Snow, David A. (2004). Social Movements as Challenges to Authority: Resistance to an Emerging Conceptual Hegemony. En Daniel J. Myers y Daniel M. Cress (Eds.), Authority in Contention (p. 3.25). Elsevier.

* Tarrow, Sidney (2012). El poder en movimiento: los movimientos sociales, la acción colectiva y la política. Alianza. 
Tilly, Charles (1986). The Contentious French. Harvard University Press.

\section{Cómo citar el artículo}

- Holguín Carrillo, Roberto (2020) Una caja de herramientas para medir el universo de protestas en México. Revista Digital Universitaria (RDU) Vol. 21, núm. 3 mayojunio. Dol: http://doi.org/10.22201/codeic.16076079e.2020.v21n3.a2.

Recepción: 25/10/2016. Aceptación: 10/12/2019. 